\title{
Numerical study on flow control of high altitude propeller
}

\author{
Shuili Ren ${ }^{1, a}$, Peiqing Liu ${ }^{1, b}$ and Lichuan $\mathrm{Ma}^{1, \mathrm{c}}$ \\ ${ }^{1}$ Beihang University, Beijing, China \\ aspt2011@126.com, ${ }^{\mathrm{b}}$ liupeiqing2011@126.com, ${ }^{\mathrm{C}}$ malichuan2011@126.com
}

\begin{abstract}
Keywords: Numerical study, low Reynolds number, flow control
Abstract. This paper establishes the stratosphere propeller geometry model, using Pointwise software to generate grid structured grid. The flow field of the propeller at the altitude of $\mathrm{H}=20 \mathrm{~km}$ in different wind speed and different rotate speed are simulated using the software FLUENT. The SST turbulence model and Gamma-Theta transition model are employed in the solver. Numerical calculations show that the Gamma-Theta transition model can accurately simulate the flow around the propeller at low Reynolds number, capture the laminar separation bubble on the blade surface, and calculate the aerodynamic characteristics of the propeller at higher altitude precisely. The suction control is applied to the active flow control of the propeller at low Reynolds number, and a preliminary numerical study is carried out. The numerical simulation results show that the suction control can improve the flow separation of the propeller surface to a certain extent, delay the separation bubble, expand the laminar flow region, improve the propeller under low Reynolds number aerodynamic characteristics, improve the efficiency of the propeller.
\end{abstract}

\section{Introduction}

At present, the research of air suction control technology in propeller active flow control is mainly aimed at two dimensional problems. The research of active control of three-dimensional propeller flow is very few, almost not. The main reason is that the flow of propeller is very complex, and the related experimental methods and numerical simulation methods are not mature enough, so there are great difficulties and challenges in the study.

This paper is coupled with the turbulence model by using the transition model in accurate prediction of blade surface flow under low Reynolds number separation bubble and transition on the basis of the further development of the three-dimensional flow suction exploratory study of laminar separation bubble around high altitude propeller.

The main goal is to study the application of numerical simulation in high altitude and low density at the low Reynolds number flows, and to investigate the control effect of the propeller blade surface in low Reynolds number flow around, providing a reference for further study.

\section{Geometrical parameters of propeller}

The propeller is designed for stratospheric airship with fixed point cruise height of $\mathrm{H}=20 \mathrm{~km}$, and the propeller efficiency is not less than $72 \%$. The blade element is low Reynolds number Eppler 387 airfoil. Propeller hub diameter $\mathrm{D}=6.5 \mathrm{~m}$, diameter $\mathrm{d}_{0}=1.3 \mathrm{~m}$, maximum width of the blade $\mathrm{b}_{\max }=0.516 \mathrm{~m}$ blade, blade number $\mathrm{N}_{\mathrm{b}}=3$, blade effect factor $\mathrm{AF}=62.3$, blade design installation angle is 20 degrees. The blade width curve is shown in Fig.1, and the blade thickness distribution curve is shown in Fig. 2 .

According to the geometry parameters of the stratospheric propeller, the Solidworks model software is used to generate the numerical simulation model. The three-dimensional shape of the propeller is shown in Fig.3.

\section{Computational grids and computational conditions}

Because of the twist angle of stratosphere propeller design greatly, in order to realize the CFD grid orthogonality, continuity and smoothness requirements of large curvature surface of propeller blade 
using O type grid, this truth can be used to simulate the propeller shape, to better ensure the orthogonality of the wall body fitted grid, which can a more accurate simulation of a near wall region of the blade flow (especially viscous effect). Therefore, the numerical calculation of propeller is divided into $\mathrm{O}+\mathrm{H}$ type. The upstream length of propeller calculation area is 10 times the propeller diameter, the downstream length is 20 times the propeller diameter, and the spanwise length is 10 times the propeller diameter.

The mesh is completed by Pointwise software, and the number of computational grids is 4.2 million. The overall mesh distribution in the propeller calculation domain is shown in Fig. 4. The distribution of the $\mathrm{O}$ mesh between the propeller surface mesh and the blade is shown in Fig.5.

The surface of propeller is free of slip boundary condition. The blade and blade flow can be divided into equal number of subdomains, with periodic boundary conditions in the initiation and termination of subdomain boundary alone on the boundary, which can simulate the real flow state, but also can reduce the number of grid computing resources, saving. Because the flow velocity is low, the flow field can be assumed to be incompressible flow. The boundary of the calculation area of the propeller upstream is set as the velocity inlet boundary, the boundary of the propeller periodic surface is set as a periodic boundary, and the other boundary is set at the pressure exit boundary.

In the ground inertial coordinate system, the flow around the propeller is unsteady, and the numerical solution of the flow field is quite complicated. The propeller is fixedly connected to the rotating coordinate system, can be regarded as the quasi steady flow around the propeller flow field, numerical solution of simple. Therefore, this article in the rotating coordinates and quasi steady flow control equations for numerical simulation of the flow around the propeller. The governing equations for the Reynolds averaged N-S equations, using the finite volume method to discrete the control equations, implicit solver based on the pressure, convection using two order upwind diffusion, using central difference scheme of pressure velocity coupling using Coupled algorithm, the discrete algebraic equations and solved by the Gauss-Seidel iteration method, calculation model were used turbulence models and transition model analysis.

\section{Results and discussion}

The calculation model of the $\mathrm{H}=20 \mathrm{~km}$ altitude of stratospheric airship propeller through FLUENT software. Under $\mathrm{V}_{0}=10,20 \mathrm{~m} / \mathrm{s}$ on the wind speed, the propeller rotation speed $\mathrm{n}=250,300,350 \mathrm{rpm}$ simulate the flow field, numerical calculation results and comparative analysis SST turbulence models and Gamma-Theta transition models.

The dimensionless aerodynamic coefficients of stratospheric propellers are defined under the wind system.

$$
\begin{aligned}
& C_{T}=\frac{T}{\rho r_{s}^{2} D^{4}} \\
& C_{\mathrm{P}}=\frac{\mathrm{P}}{\rho \mathrm{n}_{\mathrm{s}}^{3} \mathrm{D}^{5}} \\
& \eta=\frac{\mathrm{C}_{\mathrm{T}}}{\mathrm{C}_{\mathrm{P}}} \lambda \\
& \lambda=\frac{\mathrm{V}_{0}}{\mathrm{n}_{\mathrm{s}} \mathrm{D}}
\end{aligned}
$$

Among them, $\mathrm{T}$ is the propeller thrust, $\mathrm{C}_{\mathrm{T}}$ for propeller thrust coefficient, $\mathrm{P}$ for the power required for the propeller, $C_{p}$ is propeller power coefficient, $\eta$ for efficiency, $\lambda$ for the advance ratio, $V_{0}$ for velocity at the boundary of the entrance flow velocity, $n_{s}$ for propeller speed, $\rho=0.08803 \mathrm{~kg} / \mathrm{m}^{3}$ for atmospheric density at an altitude of $\mathrm{H}=20 \mathrm{~km}, \mathrm{D}=6.5 \mathrm{~m}$ for the diameter of a propeller. 
Fig.6 Fig.8 shows the curves obtained by numerical simulation of SST turbulence models and Gamma-Theta transition model of the propeller thrust coefficient $C_{T}$, coefficient $C_{P}$ and the efficiency of the powern .

It can be seen that the simulated calculation of the propeller thrust coefficient and efficiency of numerical values of the SST turbulence model results than calculated using the Gamma-Theta transition model is too large, the drag coefficient of relative error is $5.5 \%$, the efficiency is $4.7 \%$. The calculated results of the propeller power coefficient obtained by the SST turbulence model agree well with the numerical results of the Gamma-Theta transition model.

The aspirated area was $\mathrm{r}=0.37 \mathrm{R} \sim 0.86 \mathrm{R}$, the chord position was $30 \% \mathrm{c}$, the inspiratory width was $2.5 \% \mathrm{c}$, the inspiratory velocity was $6 \mathrm{~m} / \mathrm{s}$, and the suction angle was perpendicular to the wall in Fig.9.

Fig. 10 shows the comparison of the streamline distribution of each section of the propeller radial position from $r=0.4 \mathrm{R}$ to $\mathrm{r}=0.8 \mathrm{R}$ when no control and suction control is available. It can be seen from the figure, $r=0.4 \mathrm{R}$ apart airfoil surface bubble postponed $12 \%$ the length of the chord, and the separation bubble area and height significantly decreased; separation of $r=0.7 \mathrm{R}$ airfoil surface bubble postponed $8 \%$ airfoil chord length, separation $\mathrm{r}=0.8 \mathrm{R}$ airfoil surface bubble postponed $18 \%$ airfoil chord. Thus, inspiratory flow separation control to improve the propeller surface to a certain extent, inhibited the global development of propeller under low Reynolds number laminar separation bubble separation, reduce the area, delay the blade surface separation and transition, expand the laminar flow area of the surface, improve the propeller under low Reynolds number aerodynamic the characteristics, which makes the propeller thrust increases efficiency is increased.

\section{Conclusions}

Numerical calculations show that the Gamma-Theta transition model can accurately simulate the flow around the propeller at low Reynolds number, capture the laminar separation bubble on the blade surface, and calculate the aerodynamic characteristics of the propeller at higher altitude precisely. The suction control is applied to the active flow control of the propeller at low Reynolds number, and a preliminary numerical study is carried out. The numerical simulation results show that the suction control can improve the flow separation of the propeller surface to a certain extent, delay the separation bubble, expand the laminar flow region, improve the propeller under low Reynolds number aerodynamic characteristics, improve the efficiency of the propeller.

\section{References}

[1] Anthony Colozza.Initial Feasibility Assessment of a High Altitude Long Endurance Airship. NASA/CR-2003-212724.

[2] Balaji Jayaraman, Yongsheng Lian,Wei Shyy. Low-Reynolds Number Flow Control Using Dielectric Barrier Discharge Actuators. AIAA 2007-3974.

[3] Langtry, R.B. and Menter, F.R. Transition modeling for general CFD applications in aeronautics [J]. .AIAA Paper 2005-0522, 2005.

[4] Bryan D. McGranahan and Michael S. Selig. Surface oil flow measurements on several airfoils at low reynolds numbers[J].AIAA 2003-4067.

[5] M. Serdar Genç, ü Kaynak, Hüseyin Yapici. Performance of transition model for predicting low Re aerofoil flows without/with single and simultaneous blowing and suction. European Journal of Mechanics B/Fluids 30 (2011) 218-235.

[6] Dannenberg, R. E., and Weiberg, J. A., "Section Characteristics of a 10.5-Percent Thick Airfoil with Area Suction as Affected by Chordwise Distribution of Permeability,"NASA TN-2847,1952. 
[7] Genç, M.S., Kaynak, Ü., Control of laminar separation bubble over a NACA2415 aerofoil at low Re transitional flow using blowing/Suction[J]. 3th International Conference on Aerospace Sciences \& Aviation Technology, ASAT 2009.

[8] Fluent Incorporated, Fluent (V12.1.2) User's Guides, 2009.

[9] F.R. Menter, R.B.Langtry, S.R. Likki, Y.B. Suzen,P.G. Huang. A correlation-based transition model using local variables part II-Test cases and industrial applications[J]. GT2004-53454.

[10] F.R. Menter, R.B. Langtry, S.R. Likki, Y.B. Suzen, P.G. Huang. A correlation-based transition model using local variables part I-Model formulation[J].GT2004-53452.

[11] Ying Zhou,Z.J. Wang.Effects of Surface Roughness on Laminar Separation Bubble over a Wing at a Low-Reynolds Number.AIAA 2011-736.

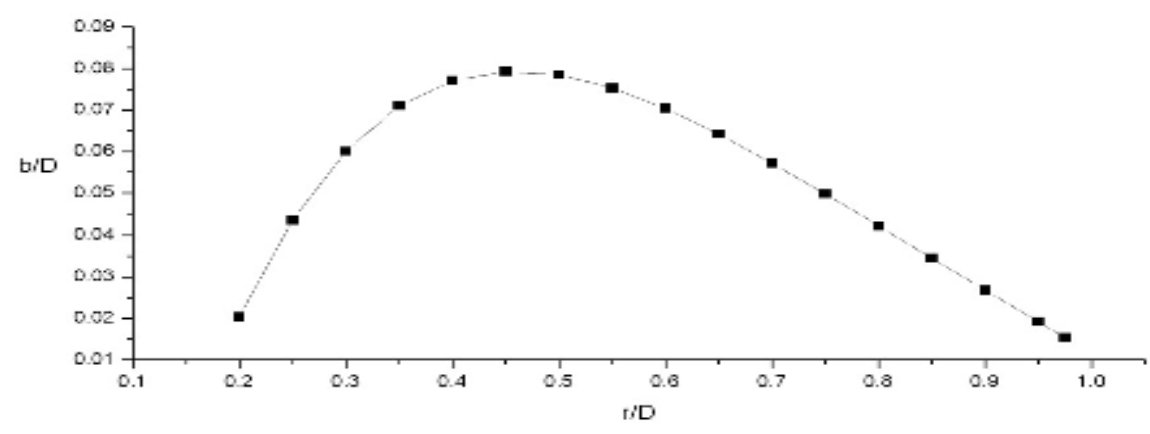

Fig.1 Radial width distribution of propeller airfoil

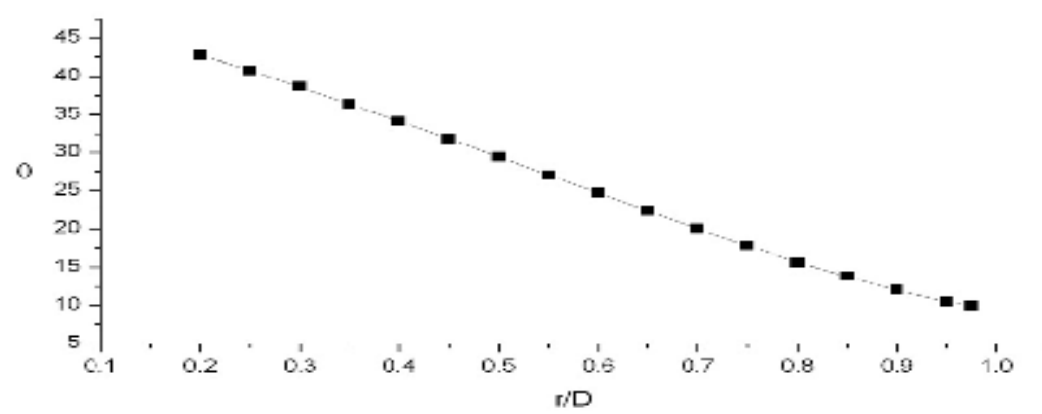

Fig.2 The blade angle of propeller is distributed along the radial direction 


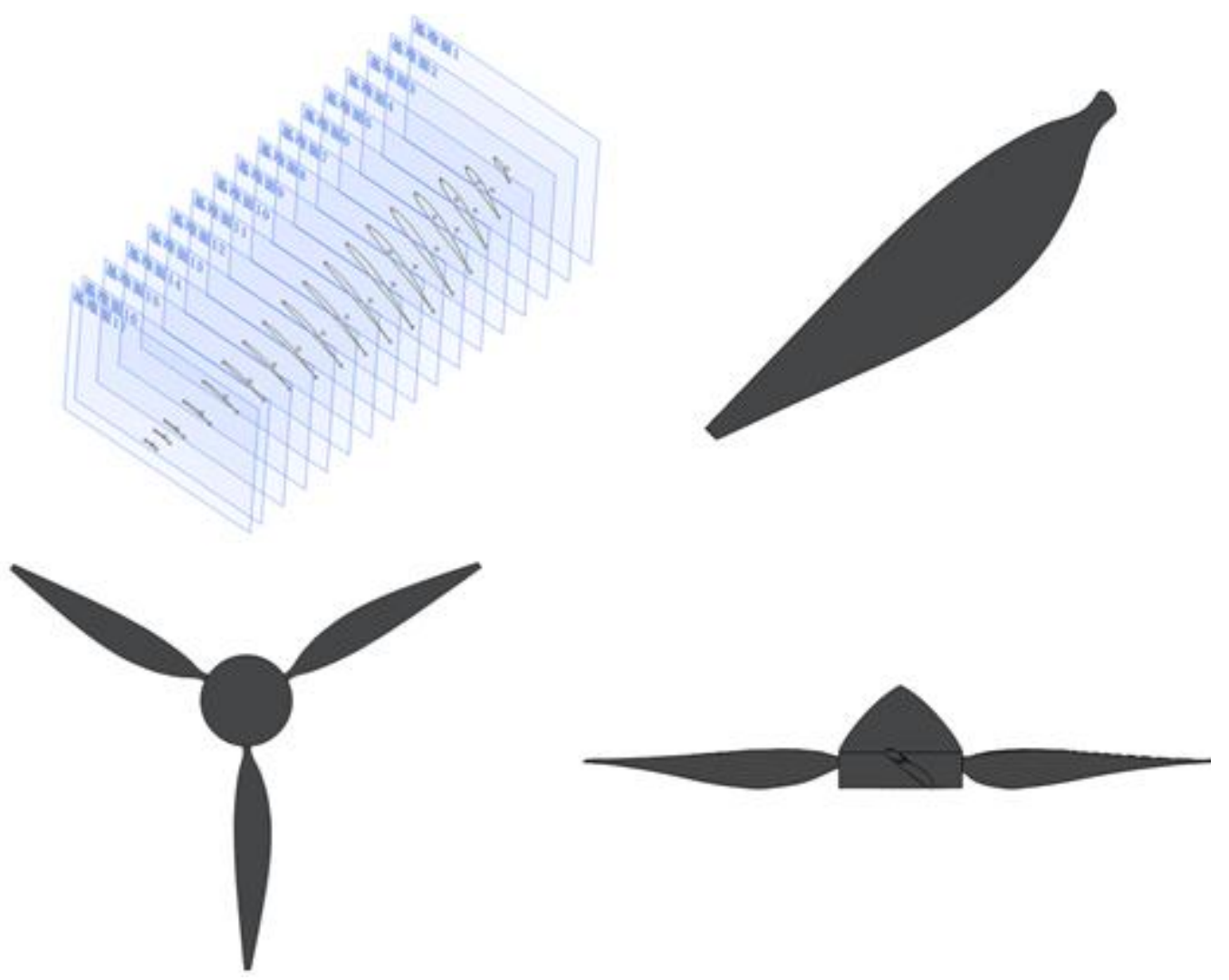

Fig.3 Schematic shape of stratospheric three blade propeller blade
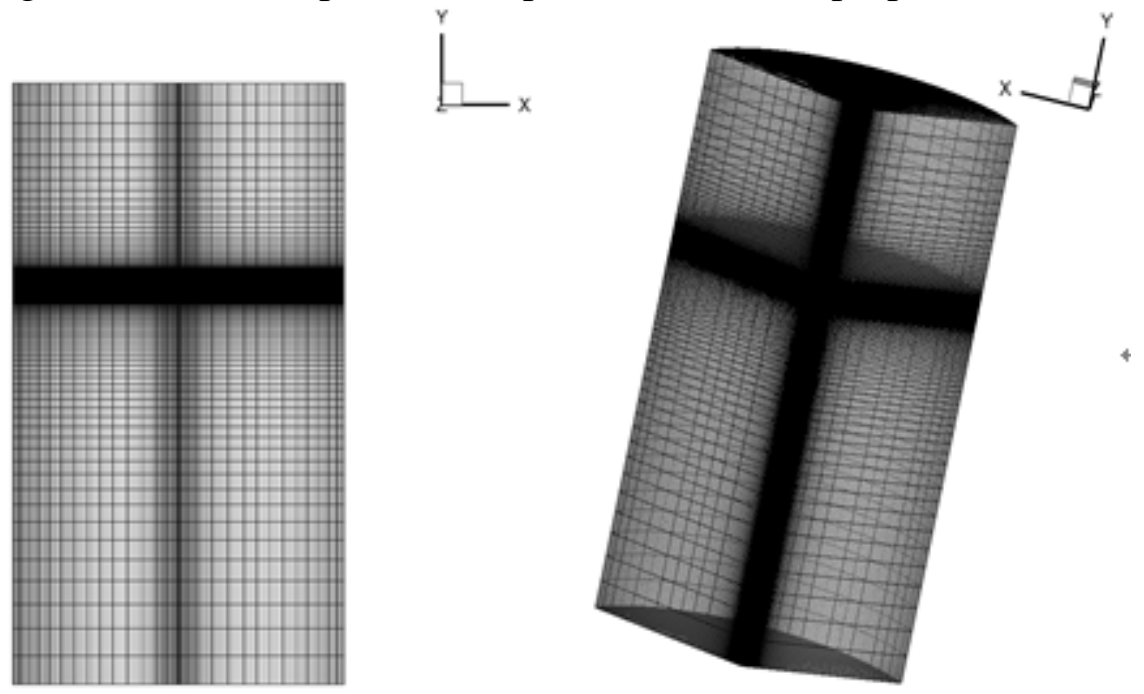

Fig.4 Computational domain integral mesh
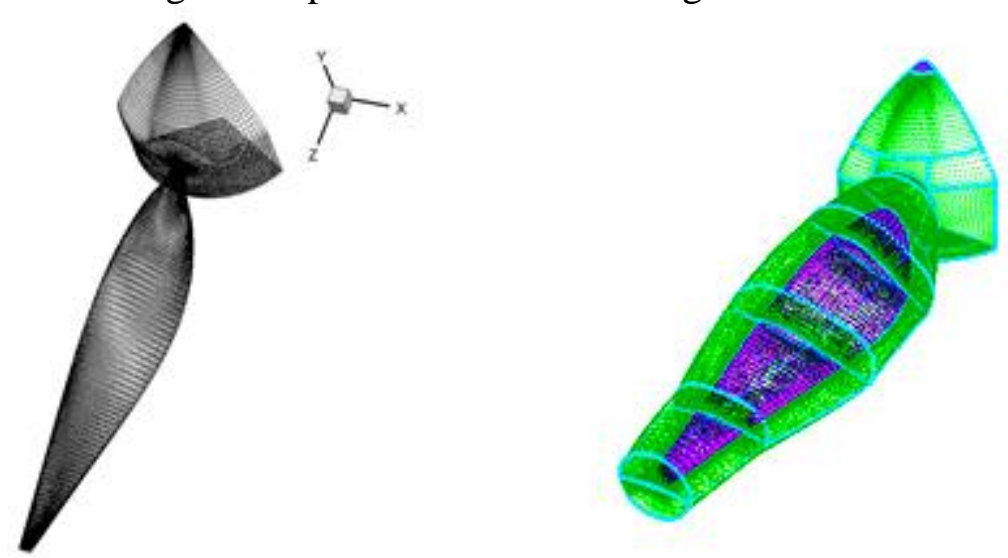

Fig.5 $\mathrm{O}$ mesh of propeller surface mesh and blade 


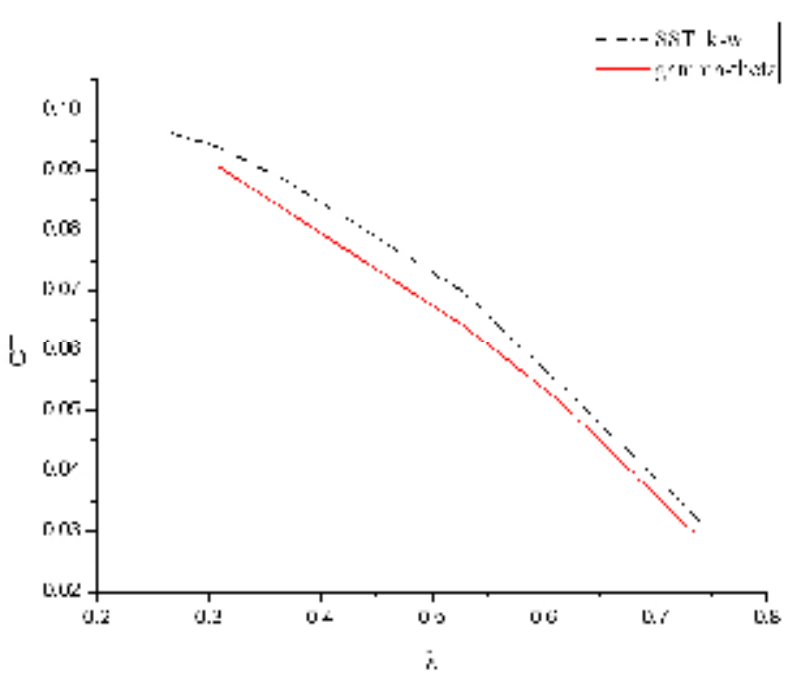

Fig.6 The $\mathrm{C}_{\mathrm{T}}$ curve of Propeller

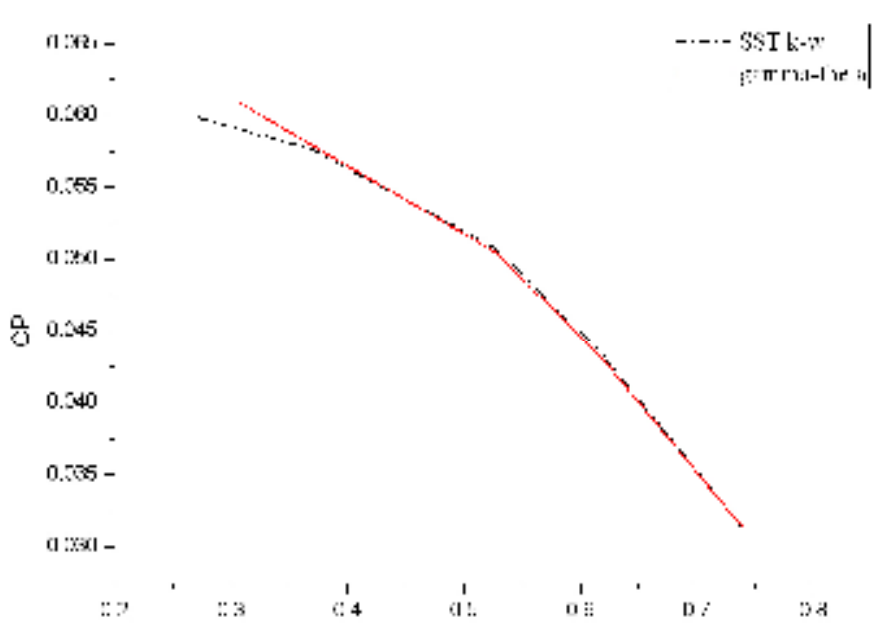

Fig.7 The $\mathrm{C}_{\mathrm{p}}$ curve of Propeller

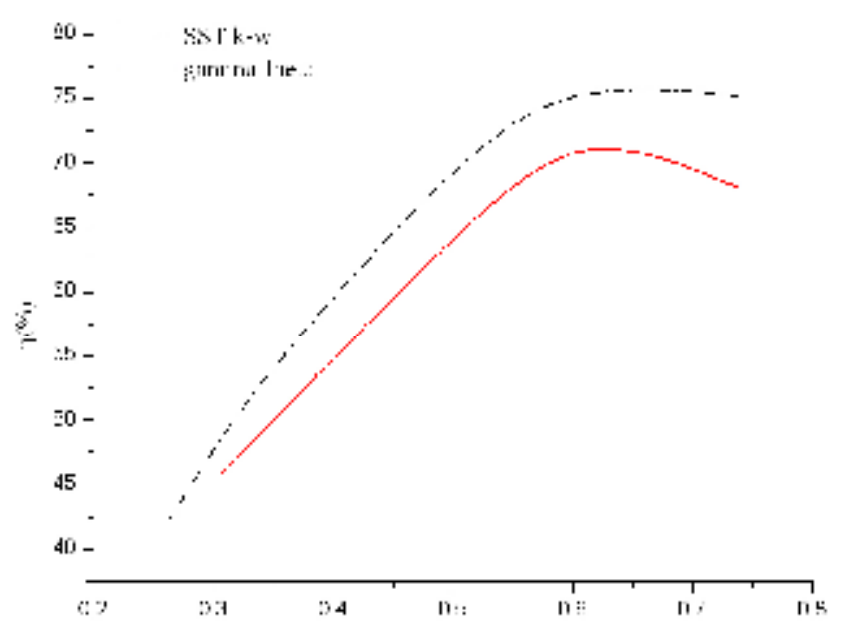

Fig.8 The $\eta$ curve of Propeller 


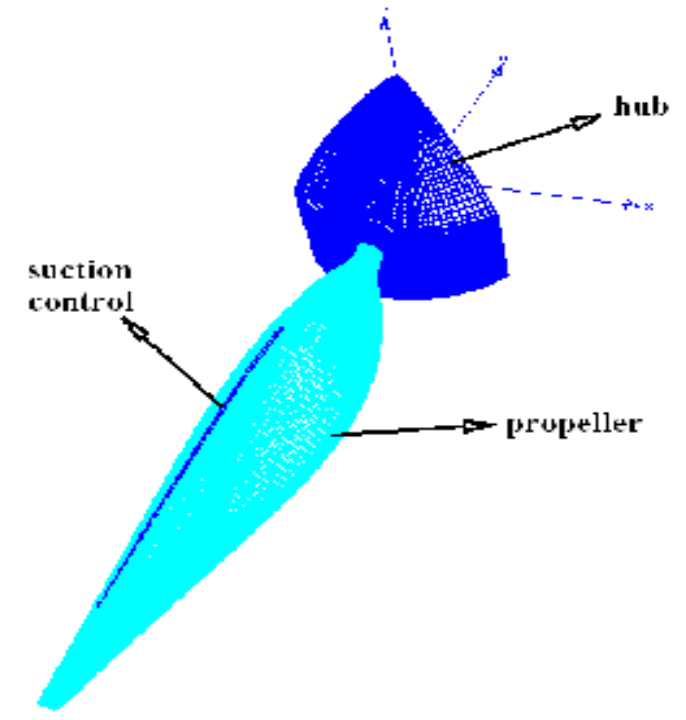

Fig.9 Schematic diagram of active control of propeller suction

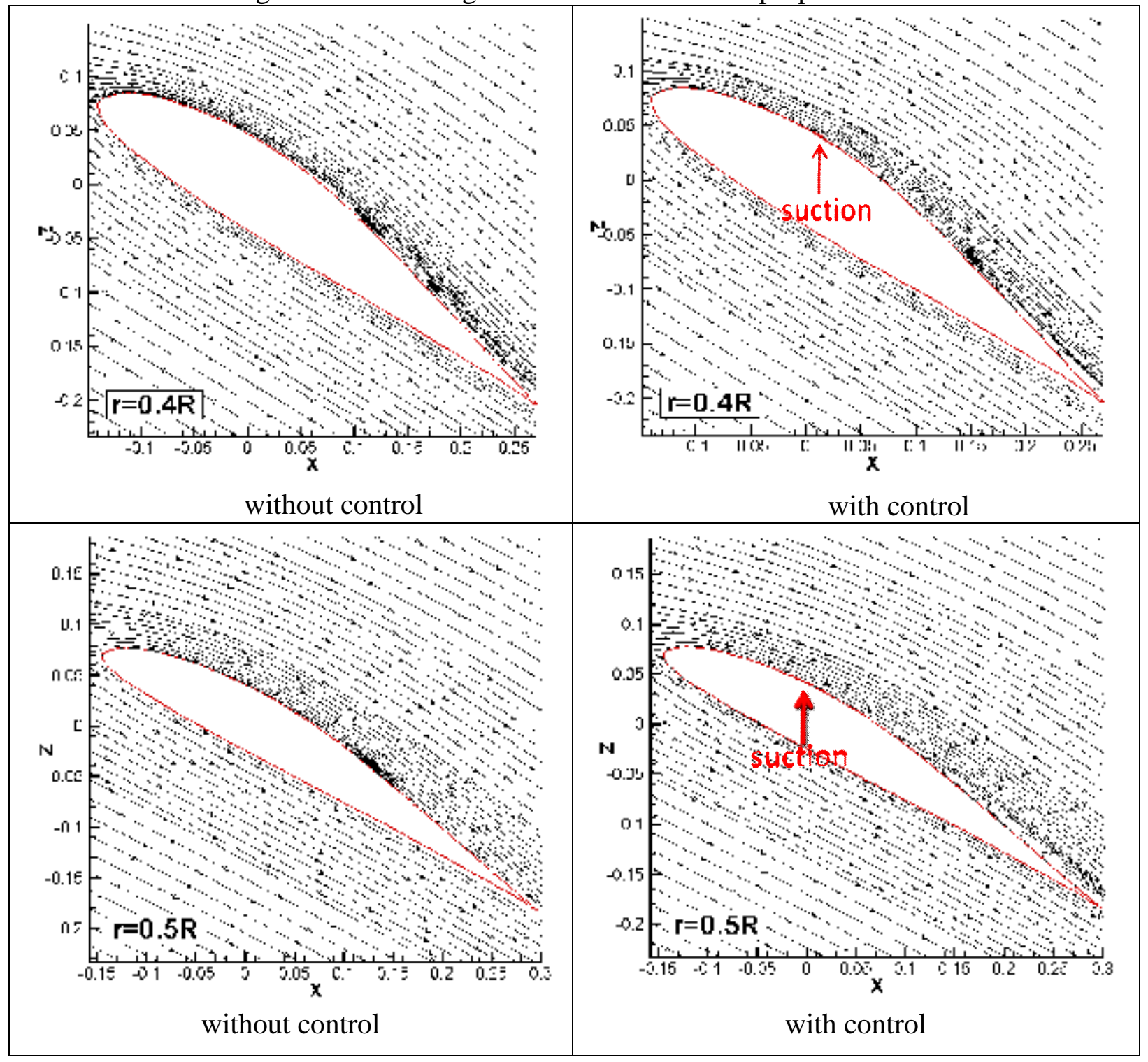




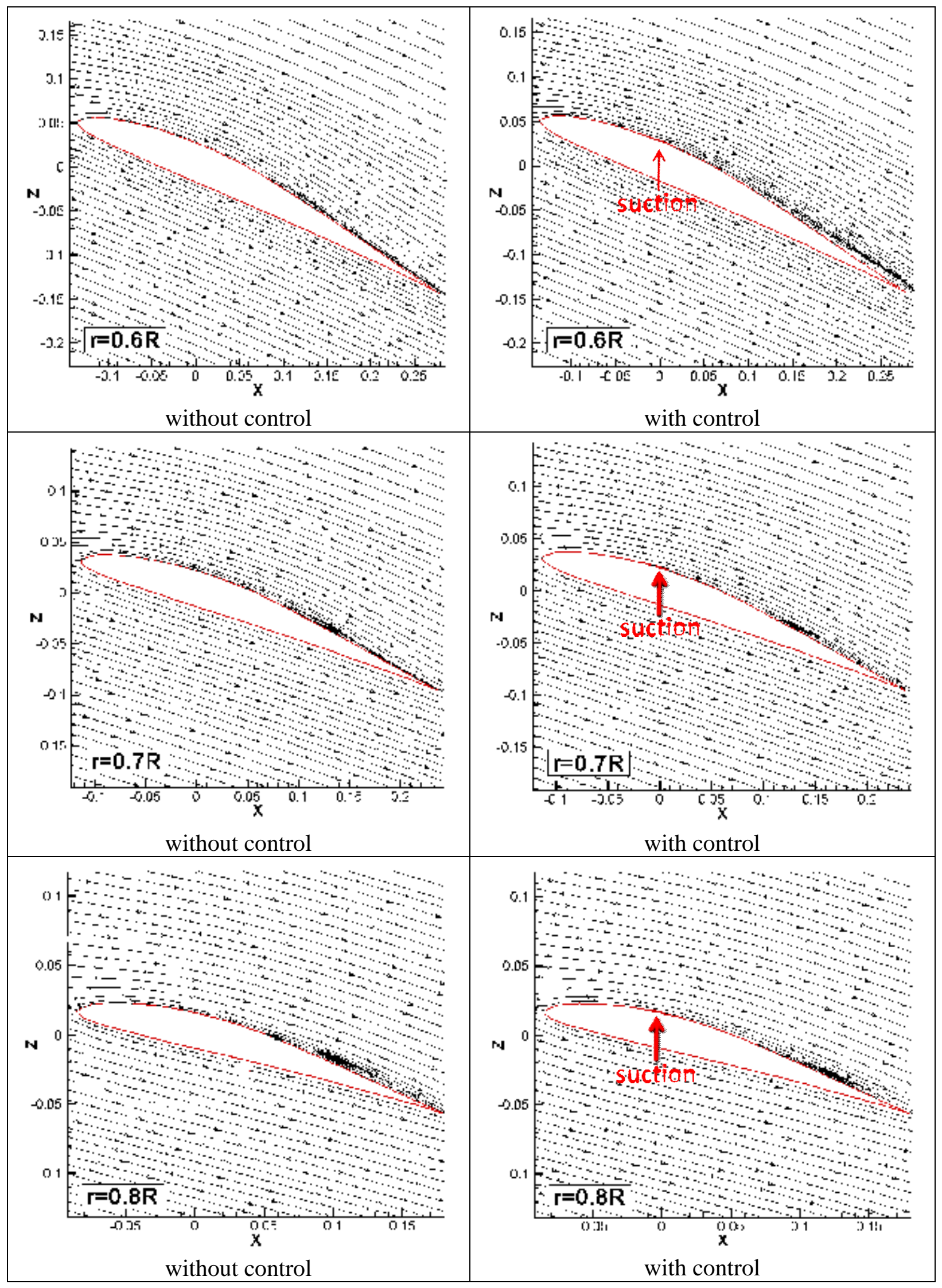

Fig.10 Comparison of streamline distribution between with or without control 